\title{
ON THE SCALAR CURVATURE AND SECTIONAL CURVATURES OF A KAEHLER SUBMANIFOLD
}

\author{
BANG-YEN CHEN AND KOICHI OGIUE ${ }^{1}$
}

Abstract. For a Kaehler submanifold of a complex space form, pinching for scalar curvature implies pinching for sectional curvatures.

1. Statement of result. The scalar curvature is, by definition, the sum of Ricci curvatures with respect to an orthonormal basis of the tangent space, and the Ricci curvature is the sum of sectional curvatures. Therefore, in general, it may be very difficult to expect some implications from the scalar curvature to the sectional curvature. However, we shall show in this note that for a Kaehler submanifold of a complex space form, pinching for scalar curvature implies pinching for sectional curvatures. Precisely, we shall prove the following pointwise theorem:

THEOREM. Let $M$ be an n-dimensional Kaehler submanifold of an $(n+p)$-dimensional Kaehler manifold of constant holomorphic sectional curvature $c$. If the scalar curvature $\rho$ of $M$ satisfies $\rho \geqq n(n+1) c-a$ at $a$ point $P$, then every anti-holomorphic sectional curvature of $M$ at $P$ is $\geqq \frac{1}{4}(c-a)$.

Although we may expect some better implication under some additional assumptions (for example, the compactness of $M$ ), our theorem is proved without any global assumption.

REMARK 1. Let $\|\sigma\|$ be the length of the second fundamental form $\sigma$ of the immersion. Then we have $\rho=n(n+1) c-\|\sigma\|^{2}$ so that the assumption " $\rho \geqq n(n+1) c-a$ " is equivalent to " $\|\sigma\|^{2} \leqq a$ ".

REMARK 2. Our theorem can be considered as a complex version of Theorem 1 of [1], in the proof of which we find a minor mistake. The correction will be given in the Appendix.

2. Proof of theorem. Let $\tilde{M}_{n+p}(c)$ be an $(n+p)$-dimensional Kaehler manifold of constant holomorphic sectional curvature $c$, and let $M$ be

Received by the editors February 26, 1973.

AMS (MOS) subject classifications (1970). Primary 53B25, 53B35.

Key words and phrases. Scalar curvature, sectional curvature, Kaehler submanifold.

1 Work done while the first author was partially supported by NSF Grant-36684 and the second author was partially supported by Matsunaga Science Foundation.

(c) American Mathematical Society 1973 
an $n$-dimensional Kaehler submanifold of $\tilde{M}_{n+p}(c)$. Let $J$ (resp. $\tilde{J}$ ) be the complex structure of $M$ (resp. $\left.\tilde{M}_{n+p}(c)\right)$ and $g$ (resp. $\tilde{g}$ ) be the Kaehler metric of $M$ (resp. $\tilde{M}_{n+p}(c)$ ). We denote by $\sigma$ the second fundamental form of the immersion. Then $\sigma$ satisfies

$$
\sigma(X, Y)=\sigma(Y, X), \quad \sigma(J X, Y)=\sigma(X, J Y)=\tilde{J} \sigma(X, Y) .
$$

Let $\xi_{1}, \cdots, \xi_{p}, \tilde{J} \xi_{1}, \cdots, \tilde{J} \xi_{p}$ be an orthonormal basis of the normal space at $x \in M$. If we set, for $\alpha=1, \cdots, p$,

$$
g\left(A_{x} X, Y\right)=\tilde{g}\left(\sigma(X, Y), \xi_{x}\right), \quad g\left(A_{x} * X, Y\right)=\tilde{g}\left(\sigma(X, Y), \tilde{J} \xi_{z}\right),
$$

then $A_{1}, \cdots, A_{p}, A_{1^{*}}, \cdots, A_{p^{*}}$ are symmetric linear transformations of the tangent space $T_{x}(M)$ of $M$ at $x$. We can easily see that $A_{x} \cdot=J A_{x}$ and $J A_{\alpha}=-A_{x} J$ so that, in particular,

$$
\operatorname{tr} A_{x}=\operatorname{tr} A_{x^{*}}=0 .
$$

Let $R$ be the curvature tensor of $M$. Then the equation of Gauss is given by [2]

$$
\begin{array}{r}
g(R(X, Y) Z, W) \\
=\sum_{x=1}^{p}\left\{g\left(A_{x} X, W\right) g\left(A_{x} Y, Z\right)-g\left(A_{a} X, Z\right) g\left(A_{x} Y, W\right)\right. \\
\left.+g\left(A_{x} \cdot X, W\right) g\left(A_{x} \cdot Y, Z\right)-g\left(A_{x} \cdot X, Z\right) g\left(A_{x} \cdot Y, W\right)\right\} \\
+{ }_{1}^{1} c\{g(X, W) g(Y, Z)-g(X, Z) g(Y, W)+g(J X, W) g(J Y, Z) \\
\quad-g(J X, Z) g(J Y, W)+2 g(X, J Y) g(J Z, W)\} .
\end{array}
$$

From (3) we see that the sectional curvature $K$ of $M$ determined by orthonormal vectors $X$ and $Y$ is given by

$$
\begin{aligned}
K(X, Y)=\frac{c}{4}\{1 & \left.+3 g(X, J Y)^{2}\right\} \\
+\sum_{x=1}^{p} & \left\{g\left(A_{x} X, X\right) g\left(A_{x} Y, Y\right)-g\left(A_{x} X, Y\right)^{2}\right. \\
& \left.+g\left(A_{x} \cdot X, X\right) g\left(A_{x} \cdot Y, Y\right)-g\left(A_{x} \cdot X, Y\right)^{2}\right\},
\end{aligned}
$$

and the scalar curvature $\rho$ is given by $\rho=n(n+1) c-\|\sigma\|^{2}$, where

$$
\|\sigma\|^{2}=\sum_{x=1}^{p}\left(\operatorname{tr} A_{x}^{2}+\operatorname{tr} A_{x}^{2}\right)
$$

An anti-holomorphic section is, by definition, a plane section $\gamma$ such that $J \gamma$ is perpendicular to $\gamma$. 
Let $X$ and $Y$ be orthonormal vectors at $x$ which generate an anti-holomorphic section. Then we can choose an orthonormal basis $e_{1}, \cdots, e_{n}$, $e_{1 *}=J e_{1}, \cdots, e_{n^{*}}=J e_{n}$ of $T_{x}(M)$ in such a way that $e_{1}=X$ and $e_{2}=Y$. For $i, j=1, \cdots, n, 1^{*}, \cdots, n^{*}$, let

$$
h_{i j}^{\alpha}=g\left(A_{\alpha} e_{i}, e_{j}\right), \quad h_{i j}^{\alpha^{*}}=g\left(A_{\alpha} \cdot e_{i}, e_{j}\right) .
$$

Then from (1) we see that

$$
h_{a b}^{\alpha}=-h_{a^{*} b^{*}}^{\alpha}=h_{a b^{*}}^{\alpha^{*}}=h_{a{ }^{*} b}^{\alpha^{*}}, \quad h_{a b^{*}}^{\alpha}=h_{a^{*} b}^{\alpha}=-h_{a b}^{\alpha^{*}}=h_{a^{*} b^{*}}^{\alpha^{*}},
$$

where $a, b=1, \cdots, n$.

On the other hand, from (4) we have

$$
K(X, Y)=\frac{c}{4}+\sum_{\alpha=1}^{p}\left\{h_{11}^{\alpha} h_{22}^{\alpha}-\left(h_{12}^{\alpha}\right)^{2}+h_{11}^{\alpha^{*}} h_{22}^{\alpha^{*}}-\left(h_{12}^{\alpha^{*}}\right)^{2}\right\} .
$$

Since $\rho \geqq n(n+1)-a$ so that $\|\sigma\|^{2} \leqq a$, making use of (5) we have

$$
\begin{aligned}
& a \geqq\|\sigma\|^{2} \\
& =\sum_{\alpha=1}^{p} \sum_{i, j=1}^{2 n}\left\{\left(h_{i j}^{\alpha}\right)^{2}+\left(h_{i j}^{\alpha^{*}}\right)^{2}\right\} \\
& \geqq \sum_{\alpha=1}^{p}\left\{\left(h_{11}^{\alpha}\right)^{2}+\left(h_{22}^{\alpha}\right)^{2}+2\left(h_{12}^{\alpha}\right)^{2}+\left(h_{1 *}^{\alpha}{ }^{*}\right)^{2}+\left(h_{2}^{\alpha}{ }^{*}\right)^{2}+2\left(h_{1}^{\alpha} *_{2}\right)^{2}\right. \\
& \left.+\left(h_{11}^{\alpha^{*}}\right)^{2}+\left(h_{22}^{\alpha^{*}}\right)^{2}+2\left(h_{12}^{\alpha^{*}}\right)^{2}+\left(h_{1 * 1}^{\alpha^{*}}\right)^{2}+\left(h_{2}^{\alpha_{2}^{*}}{ }^{*}\right)^{2}+2\left(h_{1{ }^{*} 2^{*}}^{\alpha^{*}}\right)^{2}\right\} \\
& =2 \sum_{x=1}^{p}\left\{\left(h_{11}^{\alpha}\right)^{2}+\left(h_{22}^{\alpha}\right)^{2}+2\left(h_{12}^{\alpha}\right)^{2}+\left(h_{11}^{\alpha^{*}}\right)^{2}+\left(h_{22}^{\alpha^{*}}\right)^{2}+2\left(h_{12}^{\alpha^{*}}\right)^{2}\right\} \\
& \geqq 4 \sum_{\alpha=1}^{p}\left\{-h_{11}^{\alpha} h_{22}^{\alpha}+\left(h_{12}^{\alpha}\right)^{2}-h_{11}^{\alpha^{*}} h_{22}^{\alpha^{*}}+\left(h_{12}^{\alpha^{*}}\right)^{2}\right\} \text {. }
\end{aligned}
$$

This, together with (6), implies $K(X, Y) \geqq \frac{1}{4}(c-a)$, which completes the proof.

3. Appendix: correction to the proof of Theorem 1 of [1]. From line 27 of page 606 to line 12 of page 607 should read as follows:

Let $X$ and $Y$ be orthonormal vectors at $P$. We choose an orthonormal basis $e_{1}, \cdots, e_{n}, e_{n+1}, \cdots, e_{n+p}$ in such a way that $e_{1}=X, e_{2}=Y$ and $e_{n+1}$ is parallel to $H$. Then from (6) of [1] we have

$$
\left(\sum_{i} h_{i i}^{n+1}\right)^{2} \geqq(n-1)\left\{\sum_{i}\left(h_{i i}^{n+1}\right)^{2}+\sum_{i \neq j}\left(h_{i j}^{n+1}\right)^{2}+\sum_{\alpha=n \mid 2}^{n+p} \sum_{i, j}\left(h_{i j}^{\alpha}\right)^{2}-2 c\right\}
$$


(resp. >). Applying the lemma of [1] to (7), we get

$$
\begin{aligned}
2 h_{11}^{n+1} h_{22}^{n+1} & \geqq \sum_{i \neq j}\left(h_{i j}^{n+1}\right)^{2}+\sum_{\alpha=n+2}^{n+p} \sum_{i, j}\left(h_{i j}^{\alpha}\right)^{2}-2 c \\
& \geqq 2\left(h_{12}^{n+1}\right)^{2}+\sum_{\alpha=n+2}^{n+p}\left[\left(h_{11}^{\alpha}\right)^{2}+\left(h_{22}^{\alpha}\right)^{2}+2\left(h_{12}^{\alpha}\right)^{2}\right]-2 c \\
& \geqq 2\left\{\left(h_{12}^{n+1}\right)^{2}-\sum_{\alpha=n+2}^{n+p} h_{11}^{\alpha} h_{22}^{\alpha}+\sum_{\alpha=n+2}^{n+p}\left(h_{12}^{\alpha}\right)^{2}-c\right\} .
\end{aligned}
$$

Thus, the sectional curvature of $M$ at $P$ determined by $X$ and $Y$ satisfies

$$
K(X, Y)=\sum_{\alpha=n+1}^{n+p}\left\{h_{11}^{\alpha} h_{22}^{\alpha}-\left(h_{12}^{\alpha}\right)^{2}\right\}+c \geqq 0 \quad(\text { resp. }>0) .
$$

This proves the theorem.

\section{REFERENCES}

1. B.-Y. Chen and M. Okumura, Scalar curvature, inequality and submanifold, Proc. Amer. Math. Soc. 38 (1973), 605-608.

2. K. Ogiue, Differential geometry of Kaehler submanifolds, Advances in Math. (to appear).

Department of Mathematics, Michigan State University, East lansing, MICHIGAN 48823 